\title{
Profile of Human T Cell Response to Leishmanial Antigens Analysis by Immunoblotting
}

Peter C. Melby, Franklin A. Neva, and David L. Sacks*

Laboratory of Parasitic Diseases, National Institute of Allergy and Infectious Diseases, National Institutes of Health, Bethesda, Maryland 20892

\begin{abstract}
Control and resolution of leishmanial infection depends primarily on $\mathbf{T}$ cell-mediated immune mechanisms. The nature of the leishmanial antigens involved in eliciting $\mathbf{T}$ cell immunity is unknown. We have examined the pattern of peripheral blood lymphocyte responses in patients with active, healed, or subclinical leishmanial infection to fractionated leishmanial antigens using a $\mathbf{T}$ cell immunoblotting method in which nitrocellulose-bound leishmanial antigens, resolved by one or two dimensional electrophoresis, are incorporated into lymphocyte cultures.

The proliferative and IFN- $\gamma$ responses of cells from patients with healed mucosal or cutaneous leishmaniasis were remarkably heterogeneous and occurred to as many as 50-70 distinct antigens. In contrast, responses from subjects with active, nonhealing, diffuse cutaneous leishmaniasis were either absent or present to only a small number of antigens. Control and resolution of leishmaniasis, and resistance to reinfection, is therefore associated with a $T$ cell response to a large and diverse pool of parasite antigens. The method of $T$ cell immunoblotting appears to offer a powerful, rapid, and relatively simple approach to the identification of antigens involved in eliciting a $\mathbf{T}$ cell response in human leishmaniasis.
\end{abstract}

\section{Introduction}

The intracellular parasite Leishmania causes a wide spectrum of human disease. Localized cutaneous ulceration (LCL), ${ }^{1}$ caused by Leishmania major, L. tropica, or the L. mexicana or $L$. braziliensis complexes, is generally localized and heals spontaneously over time or with specific therapy $(1,2)$. Metastatic spread to distant skin occurs in diffuse cutaneous leishmaniasis (DCL), (3) which is caused by L. éthiopica in Africa and $L$. mexicana in the new world. Patients with mucosal (mucocutaneous) disease (ML) develop destructive, disfiguring lesions of the oral and/or nasopharyngeal mucosa months to years after apparent resolution of the primary skin lesion (4). These lesions, usually due to L. braziliensis, are often

Address reprint requests to Dr. David Sacks, Building 5, Room 112, National Institutes of Health, National Institute of Allergy and Infectious Diseases, 9000 Rockville Pike, Bethesda, MD 20892.

Received for publication 19 April 1988 and in revised form 7 December 1988.

1. Abbreviations used in this paper: $\mathrm{DCL}$, diffuse cutaneous leishmaniasis; DTH, delayed-type hypersensitivity; LCL, localized cutaneous leishmaniasis; LPG, lipophosphoglycan; ML, mucosal leishmaniasis; NC, nitrocellulose; SI, stimulation index; VL, visceral leishmaniasis.

The Journal of Clinical Investigation, Inc.

Volume 83, June 1989, 1868-1875 refractory to therapy, or may reoccur after successful initial treatment. Visceral leishmaniasis (VL) or Kala-azar, caused by $L$. donovani, is characterized by fever, hepatosplenomegaly, peripheral blood cytopenias, and progressive wasting, and may lead to death if untreated $(5,6)$. In areas endemic for Kalaazar, however, subclinical infection with $L$. donovani has also been shown to occur (5-7).

The host-immune response to the different clinical forms of leishmaniasis is quite varied. In the cutaneous and mucosal forms, there is generally a low-to-moderate level of antibody production and a strong cellular response as evidenced in vivo by a positive delayed-type hypersensitivity (DTH) skin test and in vitro by Leishmania specific lymphocyte proliferation (1, 2, 8). In contrast, in the face of a strong humoral immune response, there is little evidence of a specific cellular immunity in active DCL (8-10) or Kala-azar (7, 11-14). Successful treatment, however, is associated with the development of both a positive DTH skin test and in vitro lymphocyte proliferative response to leishmanial antigens $(7,13,14)$. In addition, subclinical or asymptomatic infection with $L$. donovani can result in detectable Leishmania-specific cellular immune responses $(5-7)$.

There is strong clinical and experimental evidence that resolution of leishmanial infection depends primarily on cell-mediated immune mechanisms (15-17), and recovery from infection confers immunity to reinfection $(18,19)$. The nature of the parasite antigens involved in eliciting this immunity is not known. Because discordant $B$ and $T$ cell responses to antigenic sites have been described (20-22), attempts to define these antigens with the use of immune sera, or polyclonal or monoclonal antibody reagents may not be productive. Therefore, in a disease such as leishmaniasis, it would be advantageous to directly use $\mathrm{T}$ lymphocyte populations from immune individuals to identify potentially protective antigens. We have developed a technique to look directly at the pattern of human $\mathrm{T}$ cell reactivity to individual leishmanial antigens that is similar to, and we believe simpler and more discriminatory, than that described by Young and Lamb $(23,24)$. It involves the separation of complex antigens by one- and two-dimensional PAGE, transfer of the proteins to nitrocellulose (NC), and incorporation of the NC-bound antigen into a lymphocyte stimulation assay. In this report, we have characterized the pattern of Leishmania-specific lymphocyte responses in patients with past cutaneous leishmaniasis and ML, subclinical or self-healing $L$. donovani infection, and nonhealing DCL.

\section{Methods}

Subjects. 14 patients with healed or active leishmaniasis were studied. Six subjects had healed or healing LCL (all were residents of the United States who had acquired the disease after traveling in endemic areas); one had healed ML; three had previous subclinical or self-limited $L$. donovani infection; and four had active DCL at the time of study. The 
first patient (LCL-1) had a single cutaneous lesion on his lower lip that had been acquired in Surinam. The organism was cultured and identified as L. braziliensis guyanensis. He had complete resolution after one course of pentavalent antimony therapy. A second patient (LCL-2) had a single lesion on his chin caused by $L$. brasiliensis panamensis that was acquired in Panama. It had been present for 6 wk and was nearly resolved with pentavalent antimony therapy at the time of study. Another patient (LCL-3) had a large lower extremity ulceration with adjacent subcutaneous nodules that healed slowly after two courses of antimony therapy. His infection was acquired in Peru and the organism was typed as $L$. brasiliensis brasiliensis. A fourth patient (LCL-4) acquired infection with $L$. major in Northwest Africa. She had multiple cutaneous lesions on her trunk and extremities of several months duration that responded to two courses of antimony therapy. Another patient (LCL-5) had multiple cutaneous ulcerations on his arms due to $L$. major infection acquired in Cameroon. These lesions, which had been present for $6 \mathrm{mo}$, resolved after a single course of antimony. The final patient with cutaneous disease (LCL-6) acquired L. major infection in Afghanistan. He had several small lesions on his hand that healed with antimony therapy.

The patient with ML, a native of Columbia, had extensive involvement of the nasal mucosa for $>10 \mathrm{yr}$. Leishmania parasites, presumably $L$. braziliensis braziliensis or $L$. b. panamensis, were isolated from the lesion but failed to grow in culture medium. She underwent extensive surgical debridement and reconstruction, in addition to pentavalent antimony therapy.

Two patients had a remote history of accidental laboratory inoculation of $L$. donovani. One occurred $10 \mathrm{yr}$ before study and resulted in a small subcutaneous nodule that resolved spontaneously. She was later noted to have developed a positive Leishmanin skin test. The other, which occurred 5 yr before study, developed a large subcutaneous inflammatory nodule at the site of inoculation with no clinical signs or symptoms of visceral infection. The nodule was surgically excised and no further treatment was given. The final subject resided in an area of India endemic for Kala-azar and was studied at the Rajendra Memorial Research Institute in Patna, India. He had a positive Leishmanin skin test but no history or clinical evidence of Kala-azar. Four patients with active diffuse cutaneous leishmaniasis due to $L$. mexicana mexicana were studied. Two patients were from the Dominican Republic. One (DCL-1) had a single large nodular lesion on his right ear that had been present for $\sim 10 \mathrm{yr}$. He had received no treatment. Because of the chronic nonhealing nature of the lesion and a negative Leishmanin skin test, he was felt to have an atypical form of DCL. The second patient (DCL-2) had a 25-yr history of raised, hyperpigmented, nodular lesions on his arms, legs, face, and ears. He had a negative Leishmanin skin test but positive PPD skin test, and had received no antileishmanial therapy. The other two DCL patients were from Mexico. One patient from Tobasco (DCL-3) had nodular lesions over his ears, face, chest, back, and extremities that had been present for 6 yr. Some lesions had ulcerated and some subcutaneous nodules were present on his extremities. He was unresponsive to Leishmanin skin test antigen and had received antimony treatment 2 yr before this study without clinical improvement. The last patient (DCL-4) was from Michoacan and had extensive debilitating disease consisting of nodular, noduloulcerative and plaque-like lesions over his entire body that had been present for $\sim 20 \mathrm{yr}$. He had received several courses of antimonials in the past with only transient improvement, and was Leishmanin skin test negative.

Mononuclear cells. PBMC were isolated by centrifugation of heparinized blood over lymphocyte separation medium (LSM; Organon Teknika Corp., Durham, NC). The interface cells were washed three times in RPMI 1640 (Advanced Biotechnologies, Inc., Silver Spring, MD) and stored frozen in liquid nitrogen in RPMI-1640 with $10 \%$ fetal bovine serum and $7.5 \%$ DMSO. Before use, the cells were rapidly thawed, washed three times in RPMI 1640, and resuspended in complete culture medium containing RPMI 1640 with $10 \%$ human type AB negative serum (single donor), $2 \mathrm{mM}$ glutamine, $12 \mathrm{mM}$ Hepes buffer, and $50 \mu \mathrm{g} / \mathrm{ml}$ gentamycin.
Antigens. Soluble L. donovani (SLDA) was prepared from promastigotes from an Indian strain of $L$. donovani (National Institutes of Health, Mongi strain) that were grown in Graces Insect Tissue Culture media (Gibco Laboratories, Grand Island, NY) with $20 \%$ fetal bovine serum, $2 \mathrm{mM}$ glutamine, $100 \mathrm{U} / \mathrm{ml}$ penicillin, and $100 \mu \mathrm{g} / \mathrm{ml}$ streptomycin. After $5 \mathrm{~d}$ growth, the promastigotes were washed four times in cold PBS, and resuspended in $100 \mathrm{mM}$ Tris- $\mathrm{HCl}, 1 \mathrm{mM}$ EDTA (pH 8.0) with $50 \mu \mathrm{g} / \mathrm{ml}$ leupeptin, $50 \mu \mathrm{g} / \mathrm{ml} \alpha-2$ macroglobulin, and $1.6 \mu \mathrm{M}$ PMSF. The promastigotes were then sonicated with a Sonic disrupter (Tekmar Co., Cincinnati, $\mathrm{OH}$ ), centrifuged at $27,000 \mathrm{~g}$ for $20 \mathrm{~min}$, and the supernate was recentrifuged at $100,000 \mathrm{~g}$ for $4 \mathrm{~h}$. The supernate was then dialyzed against PBS, the soluble antigen filter was sterilized, and the protein concentration was determined. Aliquots of antigen were stored at $-70 \mathrm{C}$ and were used in lymphocyte culture at a final concentration of $20 \mu \mathrm{g} / \mathrm{ml}$. L. major soluble antigen (World Health Organization, designation MRHO/SU/59/P) was a gift of Dr. Phillip Scott, $\mathrm{NIH}$, and was prepared in identical fashion, as previously described (25). The soluble antigen preparation was used because of its ability to induce a strong $\mathrm{T}$ cell-proliferative response, and its ease of preparation in the absence of detergents enabling it to be used directly in lymphocyte cultures.

Preparation of single dimension immunoblots. SDS-PAGE (26) was performed under reducing conditions using a $7.5-15 \%$ gradient or $12.5 \%$ gel in a standard-size electrophoresis apparatus. Antigen samples were boiled for $5 \mathrm{~min}$ in an equal volume of SDS sample buffer (125 mM Tris $\mathrm{HCl}, \mathrm{pH} 6.8,25 \%$ glycerol, $2.5 \%$ SDS, 2.5\% 2-mercaptoethanol) and $0.3-0.5 \mathrm{mg}$ of protein was applied in a 9-cm strip using a single-well stacking gel. Molecular weight markers were run with the antigen. Electrophoresis was carried out until the dye front reached $12-13 \mathrm{~cm}$ from the top of the gel and a small strip of the gel was then used for silver staining (Rapid-Ag-Ostain; ICN Radiochemicals, Irvine, CA). The remainder of the gel was washed extensively over $4 \mathrm{~h}$ in transfer buffer ( $25 \mathrm{mM}$ Tris, $192 \mathrm{mM}$ glycine, $20 \%$ methanol, $\mathrm{pH} 8.2$ ). Transfer of the proteins to nitrocellulose was accomplished using the method of Towbin (27) with a constant current of $0.2 \mathrm{~A}$ for 12-16 h. The NC was then handled aseptically, washed in PBS with $100 \mu \mathrm{g} / \mathrm{ml}$ gentamycin for $4 \mathrm{~h}$, and overlaid on a 96-well flat-bottom tissue culture plate (Costar Corp., Cambridge, MA) so that the horizontal bands of transferred protein would run parallel to the eight-well rows (short dimension) of the plate. NC discs were then punched into individual wells over half the plate using a 6-mm skin biopsy punch (BakerCummins, Division of Key Pharmaceuticals, Inc., Miami, FL) to give 12 rows of quadruplicate wells. The NC was then moved horizontally to the second half of the plate and offset slightly so that the NC that remained between the first 12 rows was punched into the second 12 rows of quadruplicate wells. In this way, the entire NC sheet was incorporated into the 96-well plate, with each antigen fraction represented in quadruplicate wells. Additional discs of blank NC (blotted from a blank portion of the gel) were punched into wells for the appropriate antigen controls.

Preparation of two-dimensional immunoblots. Two-dimensional gel electrophoresis consisting of isoelectric focusing (IEF) over a pH range of 4.5-7.0 in the first dimension and SDS-PAGE in the second dimension was carried out according to the method of Hochstrasser et al. (28). SLDA in PBS was dialyzed against deionized water, lyophilized, and solubilized in IEF sample buffer (8.1 M urea, $1 \%$ SDS. $3.6 \%$ 3-[(3-cholamidopropyl)dimethylammonio]-1-propanesulfonate, $1.2 \%$ DTT, 5\% Ampholytes 3.5-10 [LKB Instruments, Inc., Gaithersburg, MD]). 25-50 $\mu$ g of SLDA in $20 \mu$ l of IEF sample buffer was focused on a $1.5 \mathrm{~mm} \times 6 \mathrm{~mm}$ tube gel for $1,600 \mathrm{~V}$-h using a minitube gel isoelectric focusing apparatus (Mighty-Small II; Hoefer Scientific Instruments, San Francisco, CA). This concentration of protein showed optimal resolution without the vertical streaking frequently seen with higher protein concentrations. The tube gel was then extruded and immediately transferred to the second-dimension slab gel (standard size, $14 \times 16 \mathrm{~cm}$ ) for SDS-PAGE. The gel was then washed and electroblotted to $\mathrm{NC}$ as in the single dimension immunoblots. The finished dimensions of the two-dimensional immunoblot approximated those 
of a 96-well tissue culture plate. After washing the two-dimensional immunoblot in PBS it was stained with $0.2 \%$ Ponceau S in 3\% TCA (filter sterilized) and the background destained with sterile, deionized water. By this method, 20-30 protein spots could be visualized that could serve to reproducibly orient the immunoblot on the 96-well culture plate. The stained immunoblot was placed over a 96-well plate, the cover placed over it and the location of the stained protein spots marked on the cover. In this way, the position of proteins in each culture plate could be reproducibly correlated with their position on a replicate gel from the same electrophoresis run, silver stained by the method of Oakley et al. (29). NC discs were then punched into the 96-well plate and the strips of NC left between the punched-out rows were then punched into the wells of a second culture plate. Thus, the entire two-dimensional immunoblot was represented in the two plates, and an individual antigen, transferred from the two-dimensional gel to the NC, would be incorporated into a single well (or possibly several wells). Control wells were included as in the single-dimension immunoblots.

Antigen stimulation of lymphocytes. PBMC were cultured at 2 $\times 10^{5}$ cells per well in 96-well flat-bottom plates in $200 \mu \mathrm{l}$ of complete medium. Wells contained electrophoretically fractionated NC-bound antigen, or blank NC with or without unfractionated antigen as controls. Cultures were incubated at $37^{\circ} \mathrm{C}$ in a $95 \%$ air $/ 5 \% \mathrm{CO}_{2}$ atmosphere, and were pulsed with $1.0 \mu \mathrm{Ci}\left[{ }^{3} \mathrm{H}\right]$ thymidine per well $(6.7$ $\mathrm{Ci} / \mathrm{mmol}$; New England Nuclear, Boston, MA) $18 \mathrm{~h}$ before termination of the culture. Antigen-stimulated cells were harvested on the fifth or sixth day of culture onto glass fiber filter paper and the incorporated ${ }^{3} \mathrm{H}$ was measured with a scintillation counter (Beckman Instruments, Fullerton, CA). It was not necessary to remove the NC before harvesting. The results are expressed as the change in counts per minute $(\triangle \mathrm{cpm}$; mean counts per minute in experimental wells-mean counts per minute in control wells) or stimulation index (SI; mean counts per minute in experimental wells/mean counts per minute in control wells).

IFN- $\gamma$ production. Supernatants from antigen stimulated cells were collected just before harvesting of cells after 5-6 d of culture. IFN- $\gamma$ activity was initially tested using a cytopathic effect inhibition assay with vesicular stomatitis virus in WISH cells (Biofluids, Inc., Rockville, MD). Experimental wells were considered positive if the IFN- $\gamma$ titer was twice that of control wells. In subsequent experiments, supernatants were assayed for IFN- $\gamma$ using a double-sandwich ELISA technique (30) using a murine MAb against human IFN- $\gamma$ (Interferon Sciences, Inc., New Brunswick, NJ) bound to microtiter plates (Immulon-2; Dynatech Laboratories, Inc., Chantilly, VA), followed by 50 $\mu \mathrm{l}$ culture supernatant, a rabbit polyclonal anti-IFN- $\gamma$, and peroxidase-conjugated donkey anti-rabbit IgG (Jackson ImmunoResearch Laboratories, Inc., West Grove, PA). After addition of the peroxidase substrate (ABTS; Kirkegaard and Perry Laboratories, Inc., Gaithersburg, MD) the OD was read and the IFN- $\gamma$ concentration was determined by comparison to a human recombinant IFN- $\gamma$ (Genentech, Inc., San Francisco, CA) standard curve. Experimental wells were considered positive if an IFN- $\gamma$ concentration $\geq 1.0 \mathrm{ng} / \mathrm{ml}$ was detected. In all subjects studied, this level was at least $4 \mathrm{SD}$ above the mean concentration in control wells.

\section{Results}

Electrophoretic separation of leishmanial antigens. Single dimension SDS-PAGE of a soluble leishmanial antigen preparation under reducing conditions resulted in separation of multiple proteins (Fig. 1). Under these conditions, there were relatively few proteins in the upper molecular weight range (molecular mass $>80 \mathrm{kD}$ ) and numerous small bands in the middle and lower molecular weight ranges. Two-dimensional electrophoresis, under reducing conditions, consisting of IEF on a minitube gel in the first dimension, and SDS-PAGE in the

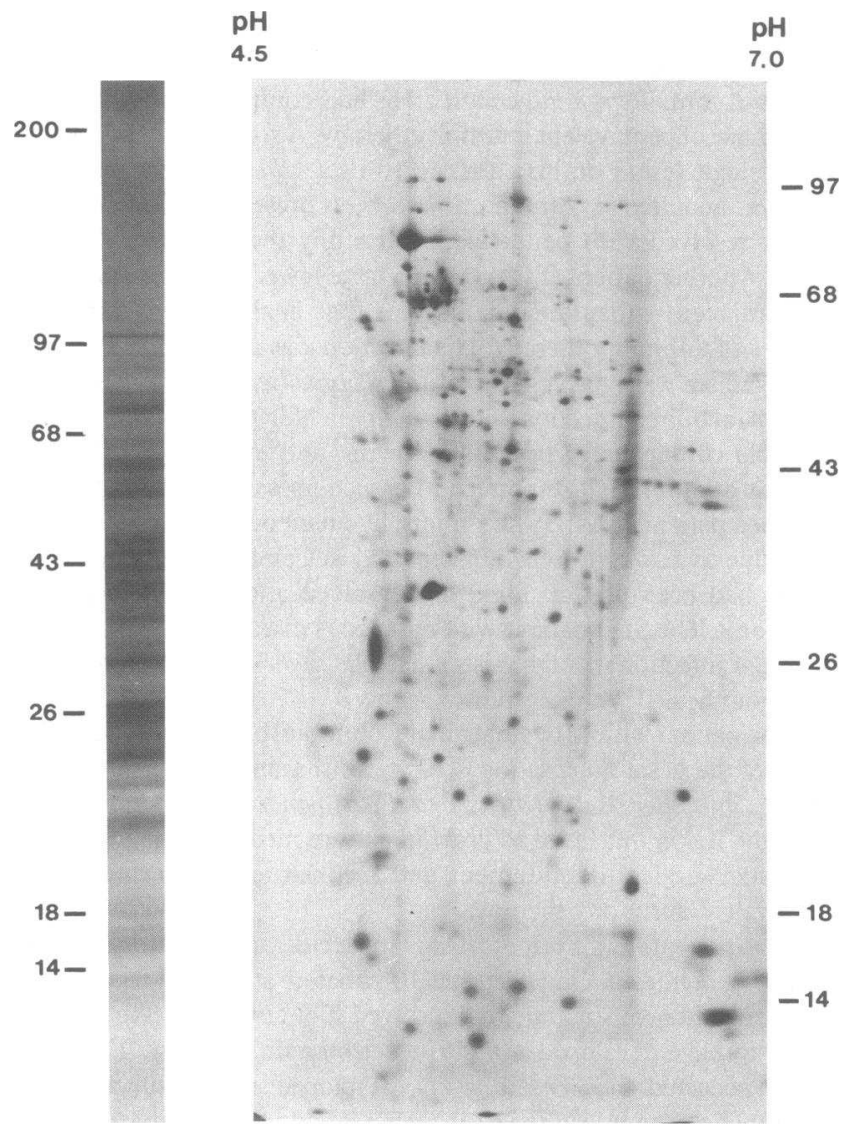

Figure 1. Silver stain of $300 \mu \mathrm{g}$ of SLDA separated by $7.5-15 \%$ gradient SDS-PAGE (left) and $25 \mu \mathrm{g}$ of SLDA separated by two-dimensional electrophoresis (right). Corresponding molecular weight markers are noted for each gel.

second dimension, resolved SLDA into $\sim 200$ discrete proteins (Fig. 1). The majority of proteins migrated within a $\mathrm{pH}$ range of 5.0-6.5.

Proliferative and IFN- $\gamma$ responses of PBMC to electrophoretically separated, NC-bound antigen. The proliferative responses of PBMC to soluble leishmanial antigens separated by one-dimensional SDS-PAGE and electroblotted on NC are shown in Fig. 2. Cells from donors who had no history of leishmaniasis and no travel within an area endemic for leishmaniasis showed no proliferative response to leishmanial antigens above background levels (Fig. 2, $a$ and $b$ ).

The pattern of lymphoproliferative response among cutaneous and mucosal patients was remarkably heterogeneous (Fig. 2, $c-f$ ). Among cutaneous patients, reactivity was strong and diffuse, more pronounced to antigen fractions of molecular masses of $60 \mathrm{kD}$ or less. Cells from the patient with $\mathrm{ML}$ responded to virtually every antigen fraction and the level of response in general was much greater than that seen in the cutaneous patients. In both instances, peak proliferative responses were consistently seen in fractions with very low molecular weight antigens (10-20 kD). There was no obvious difference in the pattern of proliferative response of cells from one cutaneous patient tested with homologous (L. major) and heterologous ( $L$. donovani) antigens (Fig. 2, $e$ and $f$ ). A large proportion of the lymphocyte-proliferative response to unfractionated soluble antigen was seen in the responses to many 

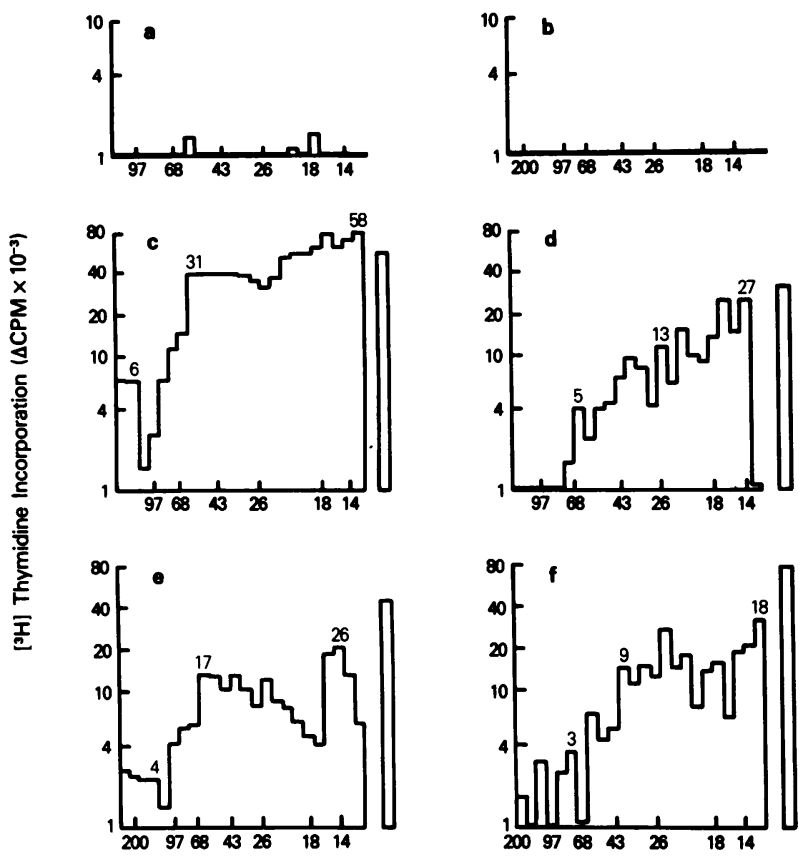

Figure 2. Profile of proliferative responses of PBMC from normal donors and patients with healed leishmaniasis, to electrophoretically separated leishmanial antigens (SLDA unless otherwise noted). The immunoblots consisted of 24 continuous fractions (quadruplicate wells) with the relative molecular weights indicated on the horizontal axis. The mean $\Delta \mathrm{cpm}$ of each fraction is represented on the vertical axis. The numbers above the figures indicate the SI of that fraction. The single column to the right of each figure represents the proliferative response of those cells to unfractionated soluble antigen in the presence of nitrocellulose. (a) normal donor; $(b)$ normal donor; $(c)$ ML (control: 2,341 cpm); (d) LCL-1 (control: $936 \mathrm{cpm);} \mathrm{(e)} \mathrm{LCL-4}$ (control: $836 \mathrm{cpm}$ ); (f) LCL-4, L. major antigen (control: 1,884 cpm).

of the antigen fractions. Because the concentrations of individual antigen present on the immunoblot were unknown, conclusions based on the comparative magnitude of the individual responses are somewhat tenuous.

The lymphocyte-proliferative response of three patients with past $L$. donovani infection were also tested by this method (Fig. 3). In the cases with prior subclinical infections and spontaneously resolved laboratory inoculation, the proliferative response was restricted to lower molecular weight antigens. In the patient who had localized $L$. donovani infection with a subcutaneous inflammatory nodule (which did not resolve spontaneously), the response was extremely strong and hetero- geneous (similar to that of the mucosal patient). In this case, the peak response also occurred in the low molecular weight ranges.

The patterns of the proliferative and IFN- $\gamma$ responses of lymphocytes to SLDA in two-dimensional immunoblots are shown in Figs. 4, 5, and 6. By using a small size two-dimensional format, the entire immunoblot could be incorporated into two 96-well culture plates, limiting the number of cells necessary for a single assay. Because of the large number of leishmanial antigens resolved by this procedure, spatial separation of antigens was somewhat limited, and in some instances there may have been more than one $\mathrm{T}$ cell antigen localized within a single well. In addition, the proliferative response to a single antigen may have overlapped into more than one well, as seen in our studies with $\mathrm{T}$ cell clones. ${ }^{2}$ Despite these limitations, two-dimensional immunoblotting resolved the heterogeneity of the response in considerably more detail. In many instances, there were responses to multiple antigens having the same relative molecular mass; evidence that the response seen within a single fraction of the one-dimensional immunoblot was directed toward multiple antigens. By using replicate gels from the same electrophoresis run and staining the NC-bound antigens before incorporation into the lymphocyte proliferation assay, we could accurately and reproducibly locate the stained protein antigens associated with a proliferative response. In some instances (especially with cells from the patients with old world cutaneous leishmaniasis) a proliferative response was present where there were no antigens visualized by silver stain of the replicate gel. These responses were consistently seen in repeat experiments, and gels from the same electrophoresis run showed an identical silver stain pattern.

Lymphocytes from normal donors, as in the single dimension analysis, showed no significant response to any region of the two-dimensional immunoblot above background levels (data not shown). Data from studies of 11 different patients (two different experiments) are shown. In the first experiment (Fig. 4), three patients having very strong Leishmania-specific proliferative responses were studied. Lymphocytes from the patients with ML showed very strong proliferative responses to many different immunoblotted antigens (approximately onethird of the culture wells were positive) with the majority of responses occurring within a $\mathrm{pI}$ range of 5.0-6.0 and peak responses occurring to antigens of $40-90 \mathrm{kD}$. Cells from the

2. Melby, P. C., and D. L. Sacks. 1988. Identification of antigens recognized by $\mathrm{T}$ cells in human leishmaniasis: analysis of $\mathrm{T}$ cell clones by immunoblotting. Manuscript submitted for publication.

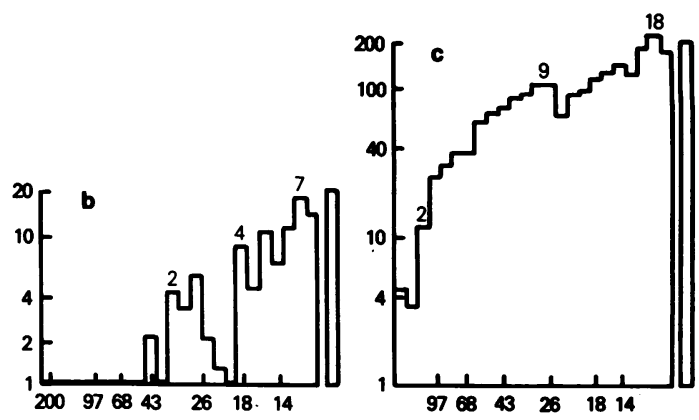

Figure 3. Profile of proliferative responses of PBMC from subjects with past $L$. donovani infection to electrophoretically separated SLDA. Data are plotted as in Fig. 2. (a) Subclinical infection from exposure in an endemic area in India (control: $1,090 \mathrm{cpm}$ ); (b) subclinical infection from accidental laboratory inoculation (control: $3,275 \mathrm{cpm})$; (c) locally contained clinical infection (unfrozen cells, control: $12,868 \mathrm{cpm}$ ). 

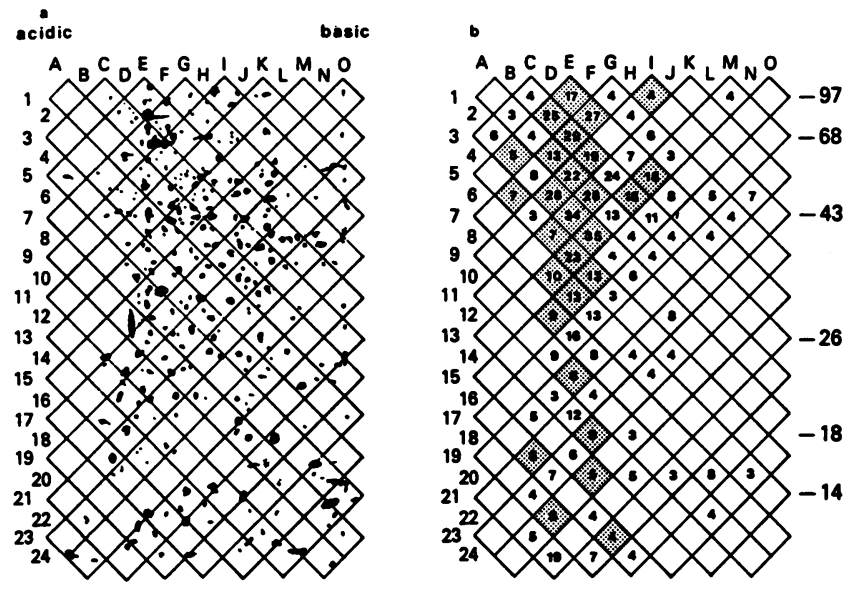

sponse to immunoblotted antigens was measured in supernatants collected from individual wells using a viral inhibition bioassay. In the three patients studied, significant IFN- $\gamma$ production was measured in $21-35 \%$ of wells having a proliferative response. In general, the level of IFN- $\gamma$ production reflected the magnitude of the proliferative response; however, there were exceptions. In some instances, a very strong proliferative response occurred without significant IFN- $\gamma$ production, and minimal proliferation was occasionally associated with a high IFN- $\gamma$ titer.

In the second experiment shown, eight different patients were studied; four had healed or resolving LCL and four had active DCL. The profiles of lymphoproliferative responses of the patients with past LCL to SLDA separated in two dimensions (Fig. 5) were also heterogeneous, though less so than those represented in Fig. 4. In general, these patients had had less extensive disease of shorter duration, and proliferative re-
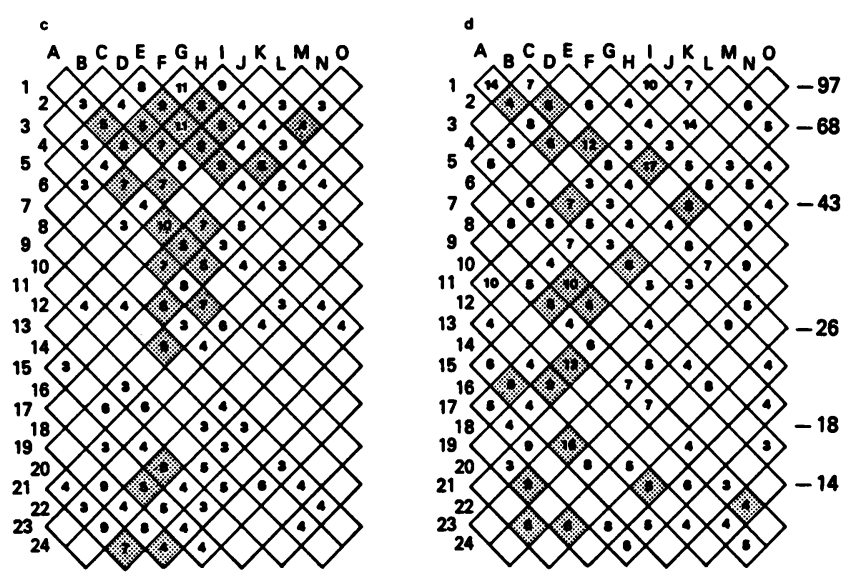

Figure 4. Profile of lymphoproliferative and IFN- $\gamma$ responses of PBMC from patients with healed ML and LCL to SLDA separated in two dimensions. The wells of the immunoblot culture plates are represented graphically with antigens having been separated by charge in the direction of the horizontal axis and by size in the direction of the vertical axis. (a) Schematic localization of individual silver-stained antigens (from a replicate gel) within the wells of the two-dimensional immunoblot. $(b-d)$ Proliferative responses of PBMNCs to an antigen or antigens within a well are recorded as the SI of that well. Only responses with a SI $>3$ are recorded. A shaded well indicates that significant IFN- $\gamma$ production was present in that well (IFN- $\gamma$ was measured in this experiment in supernatants from wells having positive proliferative responses using a viral inhibition assay). Individual patients were studied in two to four different experiments; the data shown are from replicate gels from the same experiment. (b) ML (control: $1,675 \mathrm{cpm}, \log$ IFN- $\gamma$ titer 1.6; experimental range: $5,036-5058,161 \mathrm{cpm}, \log$ IFN- $\gamma$ titers 1.9-2.4); (c) localized cutaneous $L$. donovani infection (control: $5,028 \mathrm{cpm}, \log$ IFN- $\gamma$ titer 1.6; experimental range: $15,201-15,255,583 \mathrm{cpm}, \log$ IFN- $\gamma$ titers 1.9-2.3), LCL-4 (control: $1,582 \mathrm{cpm}, \log$ IFN- $\gamma$ titer 1.0; experimental range: $5018-5027,489 \mathrm{cpm}, \log$ IFN- $\gamma$ titers 1.3-1.9).

patient with localized $L$. donovani infection responded to antigens in a pattern similar to that of the patient with ML, though the magnitude of responses was somewhat less. The patient with old world cutaneous leishmaniasis showed an equally heterogeneous but different pattern of response with more responses seen to antigens at the extremes of the $\mathrm{pH}$ gradient. Overall, $\sim 40 \%$ of the individual proliferative responses were shared among these three patients. In this experiment, IFN- $\gamma$ production by lymphocytes proliferating in re-
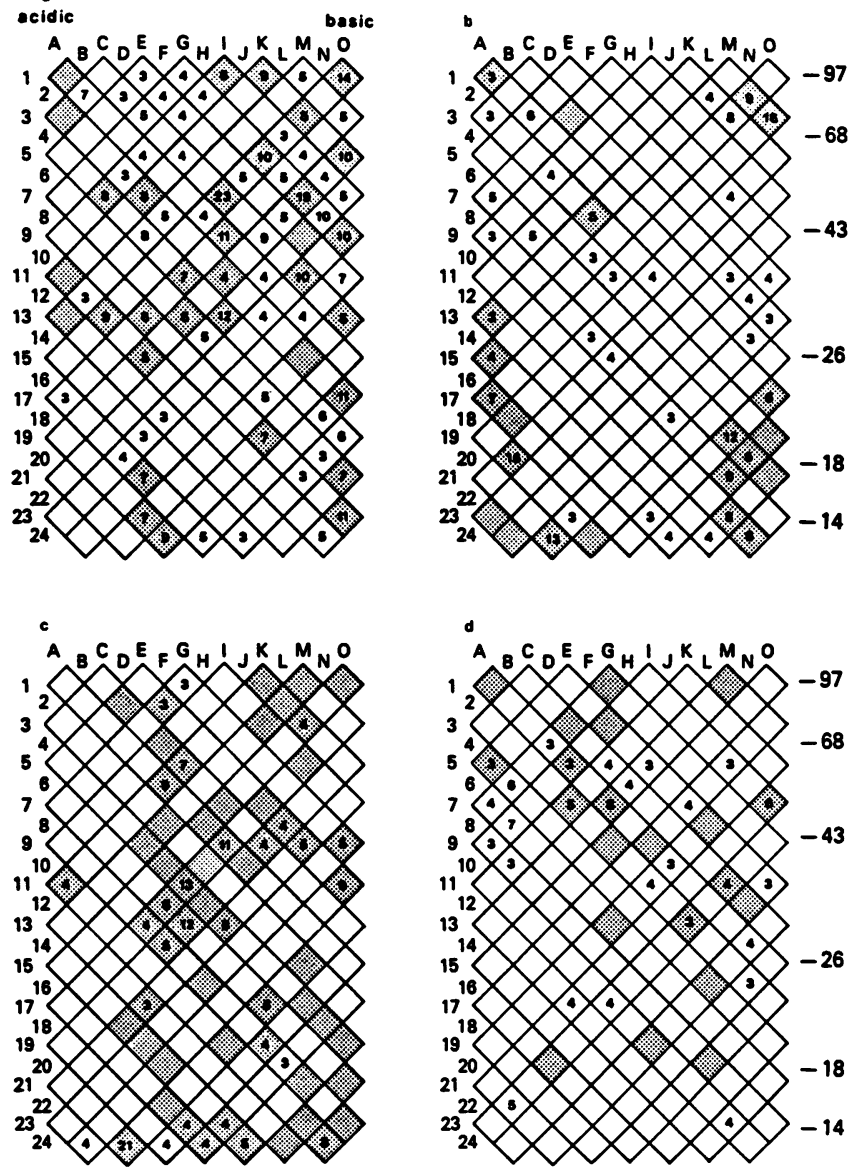

Figure 5. Profile of lymphoproliferative and IFN- $\gamma$ responses of PBMCs from patients with healed or healing LCL to SLDA separated in two dimensions. The data are presented as in Fig. 4 except that IFN- $\gamma$ production was measured in all wells using the doublesandwich ELISA technique. (a) LCL-5 (control: $488 \mathrm{cpm}$, IFN- $\gamma$ concentration $0.8 \mathrm{ng} / \mathrm{ml}$; experimental range: $1,496-11,093 \mathrm{cpm}$, IFN- $\gamma$ concentration $1.0-4.2 \mathrm{ng} / \mathrm{ml}$ ); (b) LCL-6 (control: $645 \mathrm{cpm}$, IFN- $\gamma$ concentration $0.65 \mathrm{ng} / \mathrm{ml}$; experimental range: $1,920-10,565$ cpm, IFN- $\gamma$ concentration 1.0-8.5 ng/ml); (c) LCL-3 (control: 828 cpm, IFN- $\gamma$ concentration 0.6; experimental range: $2,498-17,571$ cpm, IFN- $\gamma$ concentration $1.0->25 \mathrm{ng} / \mathrm{ml}$ ); (d) LCL-2 (control: $599 \mathrm{cpm}$, IFN- $\gamma$ concentration $<0.5 \mathrm{ng} / \mathrm{ml}$; experimental range: $1,793-4,765 \mathrm{cpm}$, IFN- $\gamma$ concentration $1.0-12.0 \mathrm{ng} / \mathrm{ml}$ ). 

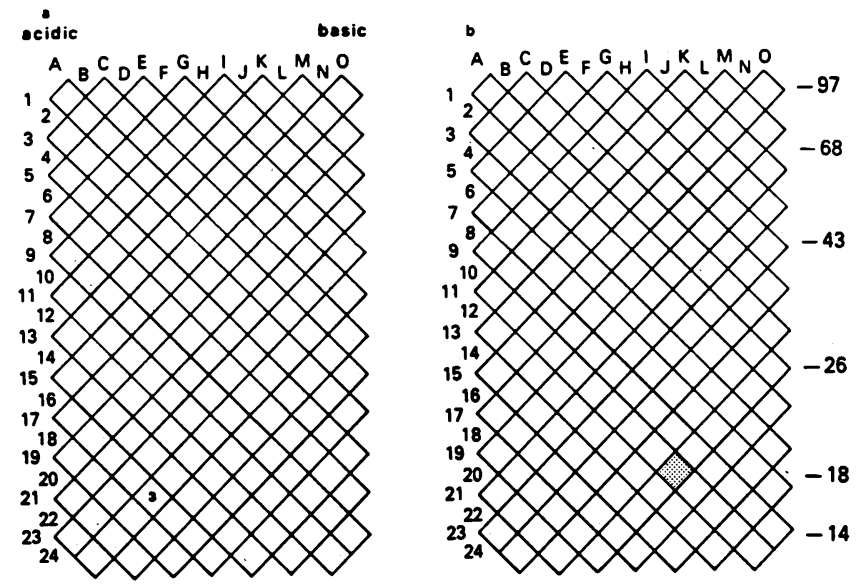

lymphokines acting on infected macrophages (15-17, 31-33). T lymphocytes from patients with active or cured leishmaniasis are reactive to complex soluble antigens of both homologous and heterologous species, but specific $\mathrm{T}$ cell antigens have not been defined. By using high-resolution two-dimensional electrophoresis techniques and measurement of antigen-specific lymphoproliferation and IFN- $\gamma$ production, we have been able to characterize the polyclonal $T$ cell response to a complex pool of parasite antigens at a level that has not been previously approached. We present evidence in this report that the Leishmania-specific $\mathrm{T}$ cell response in patients with acquired immunity (after resolution of active infection) is remarkably heterogeneous compared with nonimmune individuals. In studies of six patients with past $\mathrm{LCL}$ and one with healed ML, this heterogeneity appeared to be due to sensitization to a large and diverse pool of $\mathrm{T}$ cell antigens during infection. Many of the responses to separated, immunoblotted antigens appeared to be shared among the patients studied; the variation in the patterns of response may be due to antigenic differences among the different infecting (and sensitizing) Leishmania species, differences in the level of intensity (extent and duration) of infection, or genetic restriction of the host antileishmanial $T$ cell response. In contrast to this pattern seen in immune individuals, $T$ cells from patients with active DCL responded to an extremely limited number of separated antigens. Because these individuals have progressive, nonhealing infections, it would appear that these isolated responses are insufficient to promote resolution of infection. In addition, this paucity of responses suggests that $T$ cell reactivity cannot be uncovered by the separation of antigens that might suppress $T$ cell responses from those that might be stimulatory.

The possibility that the heterogeneous $T$ cell response of immune individuals results from recognition of a fewer number of repetitive or cross-reactive epitopes, or that protein degradation during sample preparation and electrophoresis contributes to the heterogeneity of the response cannot be excluded. That the proliferative response of $T$ cell clones could be localized to a highly restricted region of a two-dimensional immunoblot ${ }^{2}$ would argue against these possibilities. The presence of proliferative and IFN- $\gamma$ responses in areas of the immunoblots where there was no stainable protein suggests responsiveness to either poorly stainable proteins or to nonprotein antigens, in particular the Leishmania lipophosphoglycan (LPG). This complex surface molecule has been postulated to be a $T$ cell antigen because of its ability to immunize mice against cutaneous leishmaniasis $(34,35)$, though direct evidence of $\mathrm{T}$ cell stimulation has not been reported. In our own unpublished studies, we found that $\mathrm{T}$ cells from cutaneous patients responded poorly to purified LPG free of protein contaminants, which argues against the possibility that the observed heterogeneity was due to the presence of LPG dispersed throughout the immunoblot.

The presence of this remarkably heterogeneous $T$ cell response in immune individuals, in the absence of any obvious immunodominant antigen, raises issues concerning the identification of protective $T$ cell antigens. Because these individual $T$ cell responses arise from sensitization during the course of active infection, it is possible that each of these antigens might contribute in part to the control and resolution of infection, and to the immunity to reinfection that these patients acquire $(18,19)$. Identification of antigens that stimulate IFN- $\gamma$ production are further predictive of those antigens that 
might be involved in immunity. In experimental models, lymphokine production, specifically IFN- $\gamma$ production, is associated with activation of macrophages and killing of intracellular parasites (31-33). Antigen-stimulated lymphocytes from patients with cutaneous and mucosal leishmaniasis also produce IFN- $\gamma$, which can then activate macrophages and effect intracellular killing (2). In addition, cure of active visceral disease is associated with restoration of $T$ cell responsiveness and IFN- $\gamma$ production (36); and resistance to reinfection (18).

In our two-dimensional immunoblot studies, IFN- $\gamma$ production, when measured by the ELISA technique, was frequently detectable in the absence of a significant proliferative response. This is likely the result of the greater sensitivity of the ELISA, and possibly the inefficient harvest of cells from the NC-containing wells. On the other hand, the presence of a proliferative response without IFN- $\gamma$ production, suggests that $T$ cell subsets with differing capacities to produce IFN- $\gamma$ may be present $(37,38)$. The pattern of lymphokine production can be further defined as methods for measuring production of other lymphokines become available.

The characterization of the $\mathrm{T}$ cell response of patients with past subclinical or self-resolving infection with $L$. donovani offers an approach to further identify antigens that máy be important in the development of immunity to VL. In areas endemic for Kala-azar, there appears to be a significant population who have had a subclinical or self-healing infection with L. donovani. Ho et al. (5) in Kenya, and Bodaro and coworkers (6) in Brazil, have estimated the ratio of subclinical to clinical cases to be 5:1 and 6.5:1, respectively. Sacks et al. (7) studied asymptomatic family members of patients with active Kala-azar and found that $\sim 50 \%$ showed an in vitro lymphocyte-proliferative response to soluble $L$. donovani antigen. It is presumed that this $T$ cell responsiveness is associated with protective immunity.

One-dimensional immunoblot analysis of the lymphocyte response of a patient with past subclinical infection acquired by exposure in an endemic area shows that a significant response was restricted to antigen(s) in the molecular mass range of $10-20 \mathrm{kD}$. In addition, this highly restricted pattern of response was also seen in one subject who acquired an accidental, self-healing laboratory infection with $L$. donovani. This restricted pattern of response probably reflects the low intensity of infection and $T$ cell sensitization, because the locally contained, but unresolving infection with $L$. donovani in one of our subjects resulted in profound and heterogeneous $T$ cell reactivity. These limited data suggest then that in subclinical, or very self-limited infections, $T$ cells are sensitized by a fewer number of antigens, and that the responses to these antigens may be sufficient to confer immunity. We hope in future studies to obtain sufficient numbers of cells from individuals with either cured or subclinical $L$. donovani infections to better localize the responses on two-dimensional immunoblots.

In summary, we have characterized the $\mathrm{T}$ cell response in healed LCL and ML, on the basis of both proliferative responses and IFN- $\gamma$ production, to be remarkably heterogeneous, whereas the response in active DCL patients is absent or restricted to a small number of antigens. These results provide an analysis of a polyclonal $T$ cell response in human disease at a level not previously attained, and provide a framework for the study of individual antigens involved in $\mathrm{T}$ cell immunity.

\section{Acknowledgments}

We thank Al Gam for technical assistance, Dr. Dennis Hochstrasser and Dr. Mike Harrington for their generous help with the two-dimensional electrophoresis, and Sheryl Rathke for help with the preparation of this manuscript.

\section{References}

1. Schurr, E., K. Kidane, T. Yemaneberhan, and F. Wunderlich. 1986. Cutaneous leishmaniasis in Ethiopia. I. Lymphocyte transformation and antibody titre. Trop. Med. Parasitol. 37:403-408.

2. Carvalho, E. M., W. D. Johnson, E. Barreto, P. D. Marsden, J. L. M. Costa, S. Reed, and H. Rocha. 1985. Cell mediated immunity in American cutaneous and mucosal leishmaniasis. J. Immunol. 135:4144-4148.

3. Bryceson, A. D. M. 1969. Diffuse cutaneous leishmaniasis in Ethiopia. I. The clinical and histological features of the disease. Trans. R Soc. Trop. Med. Hyg. 63:708-737.

4. Marsden, P. D. 1986. Mucosal leishmaniasis. Trans. $R$ Soc. Trop. Med. Hyg. 80:859-876.

5. Ho, M., T. K. Siongok, W. Lyerly, and D. Smith. 1982. Prevalence and disease spectrum in a new focus of visceral leishmaniasis in Kenya. Trans. $R$ Soc. Trop. Med. Hyg. 76:741-746.

6. Badaro, R., T. C. Jones, R. Lorenco, B. J. Cerf, D. Sampaio, E. M. Carvalho, H. Rocha, R. Teixeira, and W. D. Johnson. 1986. A prospective study of visceral leishmaniasis in an endemic area of Brazil. J. Infect. Dis. 154:639-649.

7. Sacks, D. L., S. L. Lal, S. N. Shrivastava, J. Blackwell, and F. A. Neva. 1987. An analysis of T cell responsiveness in Indian Kala-azar. J. Immunol. 138:908-913.

8. Castes, M., A. Agnelli, and A. J. Rondon. 1984. Mechanisms associated with immunoregulation in human American cutaneous leishmaniasis. Clin. Exp. Immunol. 57:279-286.

9. Convit, J., M. E. Pinardi, and A. Rondon. 1972. Diffuse cutaneous leishmaniasis. A disease due to an immunological defect. Trans. $R$ Soc. Trop. Med. Hyg. 66:603-610.

10. Bryceson, A. D. M. 1970. Diffuse cutaneous leishmaniasis in Ethiopia. III. Immunological studies. Trans. R Soc. Trop. Med. Hyg. 64:380-387.

11. Ho, M., D. K. Koech, D. W. Iha, and A. D. M. Bryceson. 1983. Immunosuppression in Kenyan visceral leishmaniasis. Clin. Exp. Immunol. 51:207-214.

12. Rezai, H. R., S. M. Ardekalai, G. Amirhakimi, and A. Kharazmi. 1978. Immunological features of Kala-azar. Am. J. Trop. Med. Hyg. 27:1079-1083.

13. Carvalho, E. M., R. S. Teixeirá, and W. D. Johnson. 1981. Cell mediated immunity in American visceral leishmaniasis: reversible immunosuppression during acute infection. Infect. Immun. 33:498-502.

14. Haldar, J. P., S. Ghose, K. C. Saha, and A. C. Ghose. 1983. Cell mediated immune response in Indian Kala-azar and post Kala-azar dermal leishmaniasis. Infect. Immun. 42:702-707.

15. Mauel, J., and R. Behin. 1982. Leishmaniasis: immunity, immunopathology and immunodiagnosis. In Immunology of Parasite Infections. S. Cohen and K. Warren, editors. Blackwell Scientific Publications, Oxford. 299-355.

16. Pearson, R. D., D. A. Wheeler, L. H. Harrison, and H. D. Kay. 1983. The immunobiology of leishmaniasis. Rev. Infect. Dis. 5:907927.

17. Howard, J. G. 1986. Immunological regulation and control of experimental leishmaniasis. Int. Rev. Exp. Pathol. 28:79-116.

18. Manson-Bahr, P. E. C. 1961. Immunity in Kala-azar. Trans. $R$ Soc. Trop. Med. Hyg. 55:550-555.

19. Guirges, S. Y. 1971. Natural and experimental re-infection of man with oriental sore. Ann. Trop. Med. Parasit. 65:197-205. 
20. Berzofsky, J. A., L. K. Richman, and D. J. Killon. 1979. Distinct $\mathrm{H}-2$ linked Ir genes control both antibody and $\mathrm{T}$ cells to different determinants on the same antigen, myoglobin. Proc. Natl. Acad. Sci. USA. 76:4046-4051.

21. Lamb, J. R., and N. Green. 1983. Analysis of the antigen specificity of influenza haemagglutinin immune human $\mathrm{T}$ lymphocyte clones: identification of an immunodominant region for T cells. Immunology. 50:659-666.

22. Scott, P., E. Pearce, P. Natovitz, and A. Sher. 1987. Vaccination against cutaneous leishmaniasis in a murine model. II. Immunologic properties of protective and nonprotective subfractions of a solid promastigote extract. J. Immunol. 139:3118-3125.

23. Young, D. B., and J. R. Lamb. 1986. T lymphocytes respond to solid-phase antigen: a novel approach to the molecular analysis of cellular immunity. Immunology. 59:167-171.

24. Lamb, J. R., and D. B. Young. 1987. A novel approach to the identification of T-cell epitopes in Mycobacterium tuberculosis using T-lymphocyte clones. Immunology. 60:1-5.

25. Scott, P., E. Pearce, P. Natovitz, and A. Sher. 1987. Vaccination against cutaneous leishmaniasis in a murine model. I. Induction of protective immunity with a soluble extract of promastigotes. $J$. Immunol. 139:221-227.

26. Laemmli, U. K. 1970. Cleavage of the structural proteins during the assembly of the head of bacteriophage T4. Nature (Lond.). 227:680-685.

27. Towbin, H., T. Staehelin, and J. Gordon. 1979. Electrophoretic transfer of proteins from polyacrylamide gels to nitrocellulose sheets: procedures and some application. Proc. Natl. Acad. Sci. USA. 76:4350-4354.

28. Hochstrasser, D. F., M. G. Harrington, A. C. Hochstrasser, M. J. Miller, and C. R. Merril. 1988. Methods for increasing the resolution of two-dimensional protein electrophoresis. Anal. Biochem. 173:424-435.

29. Oakley, B. R., D. R. Kirsch, and N. R. Morris. 1980. A simpli- fied ultrasensitive silver stain for detecting proteins in polyacrylamide gels. Anal. Biochem. 105:361-363.

30. Curry, R. C., P. A. Kiener, and G. L. Spitalny. 1987. A sensitive immunochemical assay for biologically active MuIFN-g. J. Immunol. Methods. 104:137-142.

31. Nacy, C. A., M. S. Meltzer, E. J. Leonard, and D. J. Wyler. 1981. Intracellular replication and lymphokine-induced destruction of Leishmania tropica in $\mathrm{C} 3 \mathrm{H} / \mathrm{HeN}$ mouse macrophages. J. Immunol. 127:2381-2386.

32. Chang, K. P., and J. W. Chiao. 1981. Cellular immunity of mice to Leishmania donovani "in vitro": lymphokine-mediated killing of intracellular parasites in macrophages. Proc. Natl. Acad. Sci. USA. 78:7083-7087.

33. Murray, H. W., H. Masur, and J. S. Keithly. 1982. Cell-mediated immune response in experimental visceral leishmaniasis. I. Correlation between resistance to Leishmania donovani and lymphokine-generating capacity. J. Immunol. 129:344-350.

34. Handman, E. and G. F. Mitchell. 1985. Immunization with Leishmania receptor for macrophages protects mice against cutaneous leishmaniasis. Proc. Natl. Acad. Sci. USA. 82:5910-5914.

35. Handman, E., M. J. McConville, and J. W. Goding. 1987. Carbohydrate antigens as possible parasite vaccines. A case for the Leishmania glycolipid. Immunol. Today. 8:181-185.

36. Carvalho, E. M., R. Badaro, S. G. Reed, T. C. Jones, and W. D. Johnson. 1985. Absence of gamma interferon and interleukin-2 production during active visceral leishmaniasis. J. Clin. Invest. 76:20662069.

37. Mosmann, T. R., H. Cherwinsky, M. W. Bond, M. A. Giedlin, and R. L. Cottman. 1986. Two types of murine helper T cell clone. 1. Definition according to profiles of lymphokine activities and secreted proteins. J. Immunol. 136:2348-2357.

38. Mosmann, T. R., and R. L. Coffman. 1987. Two types of mouse helper $\mathrm{T}$ cell clone. Implications for immune regulation. Immunol. Today. 8:223-227. 